\title{
Atlas-Based Segmentation Using Level Sets and Fuzzy Labels
}

\author{
Cybèle Ciofolo \\ IRISA / CNRS ${ }^{\star \star}$ \\ Team Visages \\ 35042 Rennes Cedex, France \\ Cybele.Ciofolo@irisa.fr
}

\begin{abstract}
We propose to segment volumetric structures with an atlasbased level set method. The classical formulation of the level set evolution force presents a stopping criterion, a directional term and a regularization term. Fuzzy labels registered from an atlas provide useful information allowing to automatically tune the respective influence of the different terms according to the desired application. This is done with a fuzzy decision system based on simple rules corresponding to an expert knowledge. Two applications are presented in details in the context of 3D brain MRI: the segmentation of white matter with the tuning of the regularization term, and the segmentation of the right hemisphere. Experimental results on the MNI Brainweb dataset and on a database of real MRI volumes are presented and discussed.
\end{abstract}

\section{Introduction}

Segmentation of anatomical structures is a critical task in medical image processing, with a large spectrum of applications that ranges from visualisation to diagnosis. For example, to obtain useful quantitative information for the study of evolutive brain diseases like multiple sclerosis, an accurate segmentation of the white matter is needed.

In such a task, the main difficulties are the non-homogeneous intensities within the same class of tissue, as well as the high complexity and large variability of the anatomical structures. Among the various surface evolution methods that have been proposed in this framework, the non-parametric ones 387] showed abilities to deal with this difficulties. In particular, the level set formalism [6] provides an efficient implementation for these approaches, with the following advantages: $(i)$ efficient numerical schemes are available, $(i i)$ topological changes are allowed, and ( $i i i)$ extension of the method to higher dimensions is easy.

However, segmentation results on anatomical areas with very high positive or negative curvatures, such as sulci or ventricle horns in brain, sometimes show inaccuracies due to the regularization term involved in the evolution force. These areas generally correspond to interfaces between different classes of tissues.

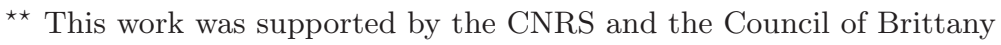


Therefore, an expert can easily locate them and select an appropriate processing. In this context, the fuzzy labels of tissue classes provided by an atlas and registered toward the processed volume are useful. However, this example can be generalized to the combination of any set of fuzzy labels and expert knowledge in order to strongly constrain the segmentation task according to the desired medical application.

Following previous work [4, we propose to integrate atlas knowledge into a level set segmentation framework 1 via a fuzzy decision system. We first focus on white matter segmentation. Fuzzy label maps adapted to any MRI volumes are created by registration and used to automatically choose the weight on curvature in the level set evolution force. Then we propose another example of level set evolution constrained to only one hemisphere with fuzzy labels.

The next section of this paper presents our methods with atlas-based preprocessing and integration of the fuzzy decision in the level set formalism. Section 3 shows our results on the MNI Brainweb dataset and on a real MRI volume database, and our conclusions and perspectives are presented in section 4 .

\section{Method}

In this section, we focus on the automatic processing set up to segment $(i)$ the white matter with a curvature constrain tuned by fuzzy labels and $(i i)$ the right hemisphere of the brain.

\subsection{A Fully Automatic Processing}

In order to achieve the segmentation of brain structures of interest in MRI volumes coming from any acquisition system, we included the segmentation algorithm in a fully automatic succession of operations. These operations are the same for each processed MRI volume. First, a brain mask and the fuzzy maps corresponding to the application are created from the data available in the MNI Brainweb dataset 5]. Then, the segmentation is performed with a level set algorithm including a fuzzy decision module. The following sections present these steps for one processed volume.

\subsection{Atlas-Based Preprocessing}

\section{Atlas Description}

The atlas we use is the MNI Brainweb dataset, which provides MRI volumes of a brain and the associated fuzzy maps. For each tissue class, the fuzzy map is a volume where each voxel has an intensity value that is proportional to its membership to this class. To segment the white matter, we use the fuzzy maps associated to the white matter, the grey matter and the cerebro-spinal fluid (CSF), as presented in the next paragraphs. For the right hemisphere application, we use only the brain tissues labeled with right laterality. 


\section{Creation of a Brain Mask}

To create an adjusted brain mask for the processed volume from the MNI Brainweb dataset, we use an affine registration algorithm (12 parameters that maximize the mutual information are computed). The grey matter, white matter and internal CSF volumes of the MNI phantom were previously combined to obtain a brain segmentation. The white matter and grey matter volumes were binary versions of the corresponding fuzzy maps, and the internal CSF was segmented with the level set algorithm described in section [1]. We then use a morphological dilation on the registration result to get a mask including the whole brain of the processed volume.

\section{Creation of the Fuzzy Label Maps}

To perform the segmentation of the white matter as precisely as possible, the evolution of the contour is constrained at the following high curvature areas:

- the interface between the grey matter and the white matter,

- the interface between the white matter and the CSF.

Concerning the segmentation of the right hemisphere, we need the distance to this hemisphere.

The fuzzy maps of the Brainweb MNI dataset are thus affinely registered to provide fuzzy maps that are adapted to the current volume.

\subsection{Integration in a Level Set Segmentation Framework}

To perform the segmentation of brain structures, the mask obtained as described in 2.2 is applied on the processed volume, and the masked data is processed with a level set algorithm.

\section{General Formulation of the Level Set Evolution Force}

In [1, the authors proposed the following region-based evolution force:

$$
F=g\left(P_{T}\right)(\rho \kappa-\nu)
$$

where $\nu$ is a constant module force, which sign leads the current interface toward the desired contour; $\kappa$ is the local curvature of the interface; $\rho$ is the weight on curvature; $g$ is a decreasing function; and $P_{T}$ is the probability of transition between the inside and the outside of the structure to be segmented. It is computed according to a preliminary classification of tissues, as described in [1]. The role of the term $g\left(P_{T}\right)$ is thus to stop the evolution of the contour at the desired location.

In previous work [4], we showed how to improve the impact of $g\left(P_{T}\right)$ with fuzzy logic. In this paper, we focus on the other parameters so that they are automatically tuned according to the application, as presented in the next paragraphs.

\section{Application to White Matter Segmentation: Design of $\rho$}

The role of $\rho$ is to regularize the contour. However, this regularization should be adapted to any part of complex volumes like the white matter. In details, it should allow the contour to propagate in very narrow protusions as the gyri. 
However, in areas corresponding to "holes" in the structure, as the ventricles, the contour should strongly contract to match the edges of the holes.

To this end, the value of $\rho$ is locally derived from the fuzzy label maps with a fuzzy decision system. This system is drawn from fuzzy control [2] and chooses appropriate weights on curvature according to a small set of rules corresponding to an expert knowledge. It is designed with the following characteristics.

The inputs are the local proportion between the grey matter and the white matter (noted GM/WM), and between the CSF and the white matter (noted $\mathrm{CSF} / \mathrm{WM}$ ). They correspond respectively to the membership of the current voxel to the interface between the grey matter and the white matter, and to the interface between the CSF and the white matter. There are two outputs of the fuzzy decision system, noted $\rho+$ and $\rho-$, which represent the weight on curvature, respectively on positive and negative curvature areas. High values of $\rho+$ and $\rho-$ respectively encourage contraction and expansion.

We use five states modeled with gaussian-like functions to represent the memberships of the fuzzy variables GM/WM, CSF/WM, $\rho+$ and $\rho-$. It is a common choice in the field of fuzzy control, which provides a partition of the universe of the fuzzy variables that is accurate enough. Our preliminary experiments showed that there was no significant change in the results with the inclusion of an offset in the design of the membership functions. Consequently, they are distributed uniformly on the universe of the variables.

Table 1. Fuzzy rules for weight on positive curvature $(\rho+)$ and negative curvature $(\rho-)$. VL: very low; L: low; M: medium; H: high; VH: very high

\begin{tabular}{|c||c|c|c|c|c|}
\hline & VL GM/WM & L GM/WM & M GM/WM & H GM/WM & VH GM/WM \\
\hline \hline $\mathrm{VL} \mathrm{CSF} / \mathrm{WM}$ & $\mathrm{VL} \rho+/ \mathrm{VH} \rho-$ & & $\mathrm{VL} \rho+$ & & $\mathrm{M} \rho+$ \\
\hline $\mathrm{L} \mathrm{CSF} / \mathrm{WM}$ & & $\mathrm{M} \rho+$ & $\mathrm{H} \rho-$ & $\mathrm{H} \rho+$ & \\
\hline $\mathrm{M} \mathrm{CSF} / \mathrm{WM}$ & & $\mathrm{H} \rho+/ \mathrm{H} \rho-$ & $\mathrm{L} \rho-$ & & \\
\hline $\mathrm{H} \mathrm{CSF} / \mathrm{WM}$ & & & & $\mathrm{VH} \rho+$ & \\
\hline $\mathrm{VH} \mathrm{CSF} / \mathrm{WM}$ & & & $\mathrm{VL} \rho-$ & & $\mathrm{VH} \rho+/ \mathrm{VL} \rho-$ \\
\hline
\end{tabular}

The fuzzy decision rules are presented in Tab. 1 They were designed according to the following principles. In areas with a high positive local curvature, as gyri, the contour should expand even if the structure is very thin. This corresponds to lower the curvature constraint in regions where the proportion of white matter is large ( VL $\rho+, \mathrm{VH} \rho-)$. On the contrary, in areas with a high negative curvature, as sulci, or in the neigbouring of ventricles, the contour should strongly contract, in order not to cross regions where the proportion of white matter is low. As this is a common problem in white matter segmentation, we take it into account by giving the priority to the proportion of CSF in the design of the rules associated to $\rho+$. This explains how Tab. 1 does not appear as a symmetric matrix. 
The associative tables between input and output fuzzy variables are computed at the beginning of the algorithm in order not to lower the speed of evolution of the level set with intermediate calculations.

Let us note that with fuzzy decision, only a few rules are needed to compute $\rho+$ and $\rho-[2]$. Thus, most of the boxes of Tab. 1 are empty, which consequently decreases the complexity of the fuzzy system. This is one of the major advantages of fuzzy logic, which avoids the use of manually-tuned parameters, and directly translates the knowledge of an expert from words to numerical decision.

\section{Application to Right Hemisphere Segmentation: Modification of the Evolution Force According to Fuzzy Labels}

This application requires a simpler system than the segmentation of white matter. As a distance map to the right hemisphere is available, the propagation of the contour can be constrained in order to minimize this distance. In details, the $\nu$ and $g\left(P_{T}\right)$ terms of the propagation force are modified until the distance is small enough (a few voxels) to use the original evolution force proposed in [1]:

- the sign of $\nu$ is chosen so that the contour propagates toward the right hemisphere,

- if the distance is large enough, $g\left(P_{T}\right)$ is set to 1 in order to increase the evolution speed.

\section{Results}

\subsection{White Matter Segmentation with Fuzzy Regularization}

\section{Results on the MNI Brainweb Dataset}

The experiments were run on the MNI Brainweb dataset [5]. After the application of a brain mask created with the combined segmentations of the white matter, grey matter and CSF, we tested the segmentation algorithm on the white matter by comparing the results with the version presented in [1], which did not involve fuzzy labels.

Let us stress that the image quality of these volumes is very good. Consequently the sulci were correctly segmented with any version of the algorithm. However, as shown on Fig. 11 the contraction of the contour was not strong enough in the occipital part of the ventricles region with the previous version, and this effect was corrected by the introduction of fuzzy labels.

The overlapping rate between our segmentation results and the MNI white matter volume was improved from $97.7 \%$ to $98.3 \%$ with the introduction of fuzzy labels, and the computation time was the same with both versions of the method.

\section{Results on 18 Real MRI Volumes}

The experiments were run on a database of $18 \mathrm{MRI}$ volumes (size $256 \times 256 \times 176$ ). It was acquired on a GE $1.5 \mathrm{~T}$ system with a sagittal orientation. The patients were all male, $35 \pm 10$ year old, healthy and right-handed persons. Let us stress 

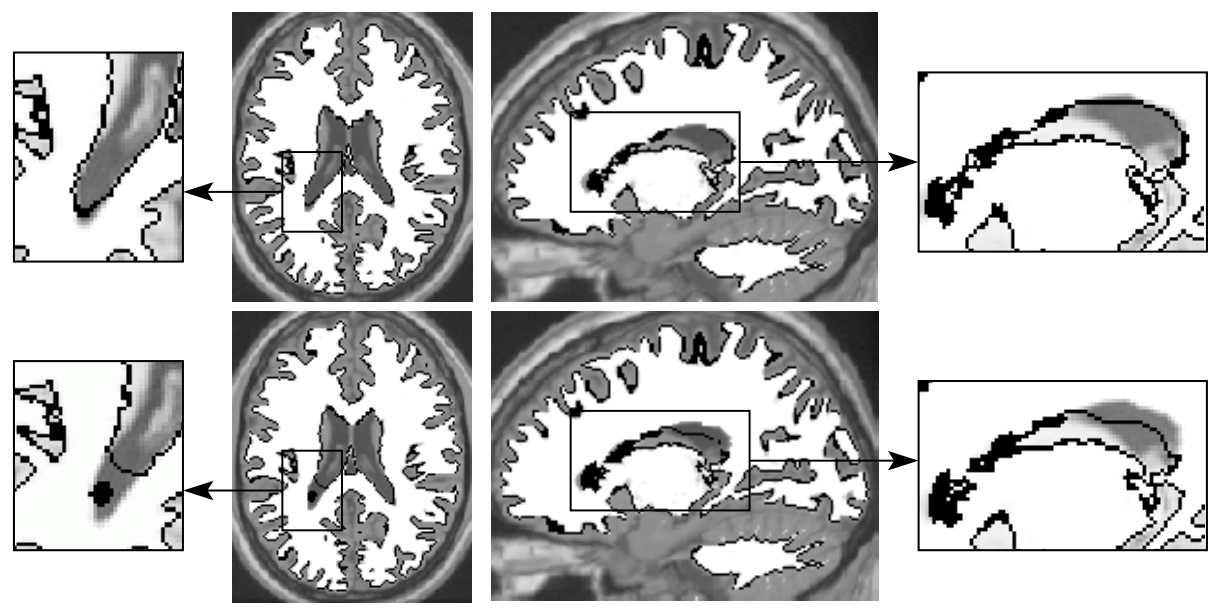

Fig. 1. White matter segmentation: the black contour shows the result with fuzzy labels (first row) and without fuzzy labels (second row), the white area corresponds to the true white matter.

that for each segmentation, we used exactly the same set of parameters for the 18 volumes.

To create adapted initial volumes, we used the registered version of the MNI brain mask that was obtained as described in 2.2, and perform a morphological erosion, as we know that the white matter is located inside the brain. The obtained volume is thus a full volume, with no hole corresponding to the location of the ventricles.

As for the previous set of experiments, we compared the segmentation results with and without the fuzzy labels. As the initial contour does not present any hole around the ventricles, its evolution in this area corresponds to a strong contraction and requires to increase the constraint on curvature. Fig. 2 shows how the introduction of fuzzy labels improved the final segmentation result. Moreover the computation time was increased by only $5 \%$ with the introduction of fuzzy labels.

For comparison, we also tested the white matter segmentation with a gradient-based level set, with the same set of parameters. The gradient-based method failed to follow the white matter borders in the areas that are too far from the initialization volume, on the MNI volume as well as on the 18 volumes database.

\subsection{Right Hemisphere Segmentation}

The experiments were run on the same database as the white matter segmentation. The level set algorithm was initialized with a parallelepipedic box located inside the brain. 


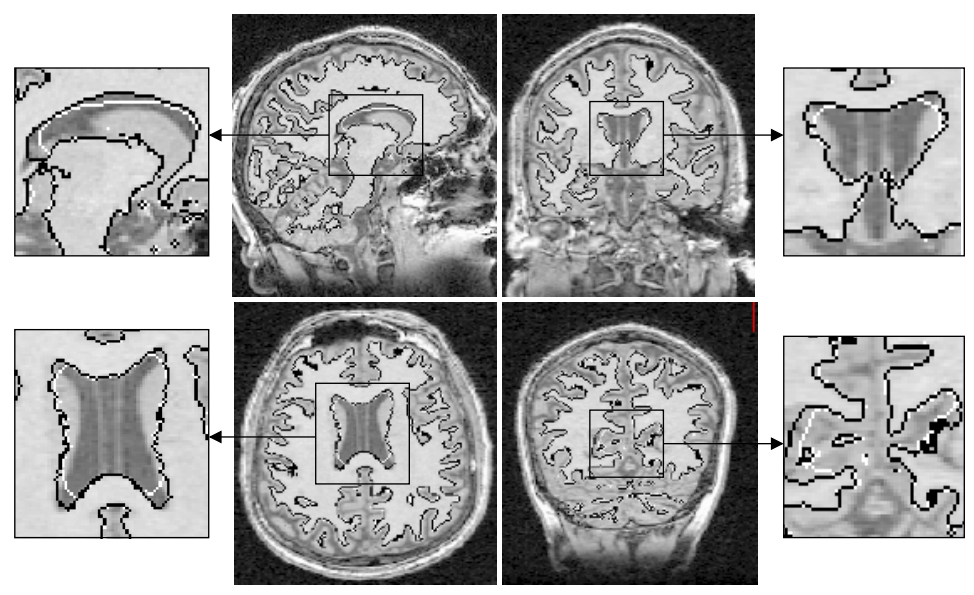

Fig. 2. White matter segmentation: the black contour shows the result with fuzzy labels and the white contour without fuzzy labels. The final contour matches the edges of ventricles and sulci with the use of fuzzy labels.
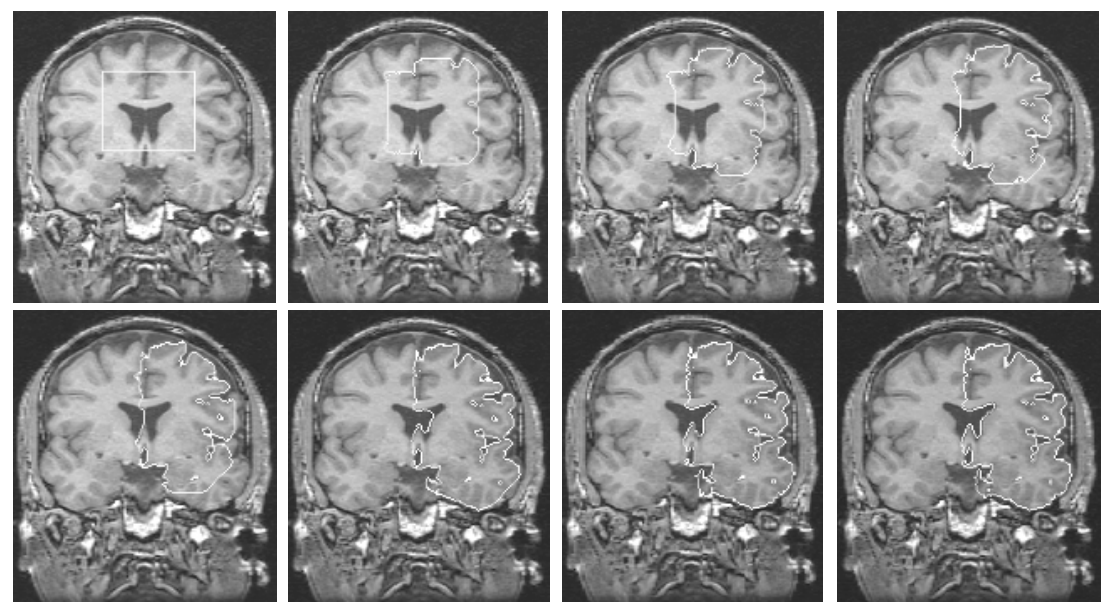

Fig. 3. Brain segmentation constrained to the right hemisphere

As soon as the contour begins to evolve, it is attracted to the right hemisphere because of the distance constraint. The evolution is shown on Fig. 3 and the priority given to the distance constraint can be observed in the ventricles area. The contour is attracted to the right hemisphere before segmenting the ventricle. It finally converges at the desired location.

This shows that the level set evolution can be constrained relatively to a given set of fuzzy labels in order to focus on any anatomical structure. 


\section{Conclusion and Future Work}

We presented an automatic method for the segmentation of volumetric structures. Fuzzy labels coming from an atlas were used to constrain the propagation of a level set contour. Two examples of label-constrained evolution forces were described: one designed for the segmentation of the white matter, the other one dedicated to the right hemisphere segmentation. In all the cases, fuzzy logic avoided the introduction of weighting parameters to balance the respective influence of the terms involved in the evolution force.

Experimental results showed that the combined fuzzy logic and level set methods were at least as robust as the original algorithm. The quality of results was improved for white matter segmentation both on the MNI Brainweb dataset and on a database of 18 volumes. Concerning the right hemisphere segmentation, the evolving contour converged rapidly to the desired structure.

This last set of experiments provides a lot of perspectives. We demonstrated that fuzzy labels could constrain the level set evolution in specific areas, while improving the accuracy of the segmentation. A variety of medical applications could thus take advantage of this technique to quantitatively assess the evolution of anatomical structures, such as lesions. Moreover, the use of several contours propagating in competition, for example one for each hemisphere, could lead to very precise segmentation results in the region of convergence.

\section{References}

1. Baillard, C., Barillot, C.: Robust 3D Segmentation of Anatomical Structures with Level Sets. Proceedings of MICCAI (2000), 236-245

2. Bouchon-Meunier, B.: La logique floue et ses applications. Addison-Wesley (1995)

3. Caselles, V., Kimmel, R., Sapiro, G.: Geodesic Active Contours. Int. Journal of Computer Vision, Vol 22 (1997) 1 61-79

4. Ciofolo, C., Barillot, C., Hellier, P.: Combining Fuzzy Logic and Level Set Methods for 3D MRI Brain Segmentation. Proceedings of the IEEE International Symposium on Biomedical Imaging (2004)

5. Collins, D. L., Zijdenbos, A.P., Kollokian, V., Sled, J.G., Kabani, N.J., Holmes, C.J., Evans, A.C.: Multimodality Imaging - Design and Construction of a Realistic Digital Brain Phantom. IEEE Transactions on Medical Imaging, Vol 173 (1998) 463-468

6. Osher, S., Sethian, J.A.: Fronts Propagating with Curvature Dependant Speed: Algorithms Based on Hamilton-Jacobi Formulation. Journal of Computational Physics, Vol 79 (1998) 12-49

7. Paragios, N.: A Variational Approach for the Segmentation of the Left Ventricle. Int. Journal of Computer Vision, Vol 50 (2002) 3 345-362

8. Yezzi Jr., A., Tsai, A., Willsky, A. A Statistical Approach to Snakes for Bimodal and Trimodal Imagery. Proceedings of the IEEE Conference on Computer Vision (1999) 